\title{
Spontaneous compartment syndrome in a patient with diabetes and statin administration: a case report
}

\author{
Stefano Flamini · Carmine Zoccali • \\ Emanuele Persi · Vittorio Calvisi
}

Received: 25 February 2007 / Accepted: 8 April 2008/Published online: 14 May 2008

(C) Springer-Verlag 2008

\begin{abstract}
Compartment syndrome is a condition characterized by pressure increasing in the inextensible muscular compartments that leads to a decrease of capillary perfusion with consequent ischemic lesions of the logia elements. The authors report a case of an unusual compartment syndrome with spontaneous onset in a patient with type II diabetes and chronic therapy with statins (Atorvastatin). The condition was successfully treated by a fasciotomy and medical support. The importance of a correct anamnesis and a high level of suspicion is emphasized.
\end{abstract}

Keywords Spontaneous compartment syndrome . Rhabdomyolysis $\cdot$ Statins $\cdot$ Diabetes microangiopathy

S. Flamini · C. Zoccali - E. Persi · V. Calvisi

Medical School of L'Aquila, "San Salvatore" Regional

Hospital, L'Aquila (AQ), Italy

e-mail: stefanoflamini@libero.it

C. Zoccali ( $₫)$

Via Monte Rotondo 8, 67100 L'Aquila, Italy

e-mail: carminezoccali@libero.it

C. Zoccali

IFO, Regina Elena Hospital, Rome, Italy

E. Persi

"Santi Filippo e Nicola" Hospital, Avezzano (AQ), Italy

e-mail: persiemanuele@interfree.it

V. Calvisi

University of L'Aquila, L'Aquila, Italy

e-mail: calvisiv@libero.it

\section{Introduction}

Compartment syndrome (CS) is a situation in which there is an increase in interstitial pressure in an osteofascial compartment causing a decrease in tissue perfusion, and ischemic lesions in the nervous and muscular structures [1]. The acute and chronic forms can be distinguished from each other.

The most frequent cause of acute compartment syndrome (ACS) is linked to traumatic injuries of both the bone and the soft tissues [2]. However, surgery can lead to ACS due to an erroneous position of the patient on the operating table, for trendelenburg, prolonged hypotension or a specific operation, such as intramedullary osteosynthesis [3, 4].

Other ethiopathogenic factors may be ischemic revascularization [5], intravenous drug abuse [6, 7] and alcohol consumption [8].

Chronic compartment syndrome (CCS) is rarer than ACS. This condition caused by intense muscular stress is typical in athletes [9]. However, other possible etiopathogenic factors, such as primitive and secondary myopathies [8-10], statin assumption [11, 12], ischemic pathologies [8] metabolic disorders (mellitus diabetes $[13,14]$ and hyperaldosteronism), are also observed.

\section{Case report}

A 46-year-old Caucasian man complained of pain bilaterally localized in the legs while at rest, which improved ten minutes after onset but then resumed thirty minutes later.

He was admitted to the Emergency Department where, in absence of other signs, he was diagnosed with bilateral sciatic pain and treated as an outpatient. 
The day after the patient returned to the hospital complaining that there was no improvement in symptomatology. On examination, the left leg was swollen with a tense elastic consistency, and compression of the anterior and posterior leg compartments caused pain.

Palpation reveled a termic gradation from the middle third of the leg down. There was hyposthenia of the extensor communis digitorum (ECD) and of the extensor proprius hallucis (EPH) and plantar foot anaesthesia.

Because of the severity of the case the patient was admitted to the orthopaedic ward for treatment.

The patient has suffered from renal failure since he was 18 years old. This is caused by an unknown inflammatory disorder. He was treated with ramipril $2.5 \mathrm{mg} / \mathrm{die}$ and acetylsalicylic acid $100 \mathrm{mg} / \mathrm{die}$, and in addition atorvastatin $10 \mathrm{mg} /$ die due to hyperlipidemia for 3 years.

Measuring the intracompartmental pressure with the Striker system revealed $97 \mathrm{mmHg}$ pressure in the anterior logia and $62 \mathrm{mmHG}$ pressure in the posterior logia. An echo-color Doppler evidenced diffused angiosclerosis, calcific atheromatous lesions with good distal hemodynamic compensation and posterior tibial artery obstruction.

The venous system was pervious.

CS was diagnosed given the simptomatology, the increasing pain and the intracompartmental pressure values, and a mini invasive fasciotomy of the posterolateral and anterolateral compartments was performed. The muscles appeared to be ischaemic and oedematous but a little improvement of EPA and ECD function and a relief of pain was already noted in theatre.

In the first postoperative day the patient complained about continuous pain in the calf and Achilles tendon medial surface. The LDH, CK-CPK, myoglobinemia, aldolase, GOT and GPT blood tests were very elevated (CPK 9,351 U/l). The next test showed an improvement in the ematochemical levels which normalized after two weeks.

Treatment consisted of intravenous support and diuretic drugs to avoid further damage to the knees. The patient was diagnosed with type-II diabetes with a blood glucose level of $148 \mathrm{mg} / \mathrm{dl}$. Hepatitis markers and drug abuse tests were negative; histopathologic examination of the muscular biopsy revealed the presence of interstitial eosinophilic granulocytes, hyalinosis of muscular fibres and these findings were compatible with the diagnosis of autoimmune, allergic or parassitic inflammatory myopathy (Figs. 1, 2).

The electrolytes ematochemical levels were normal and the self-antibody research was negative as well. Electromyography indicated a myogenic lesion.

After 15 days surgery was performed by cutaneous accesses closure. However, on the 25-postoperative day an ischemic area in the Achilles tendon medial surface was

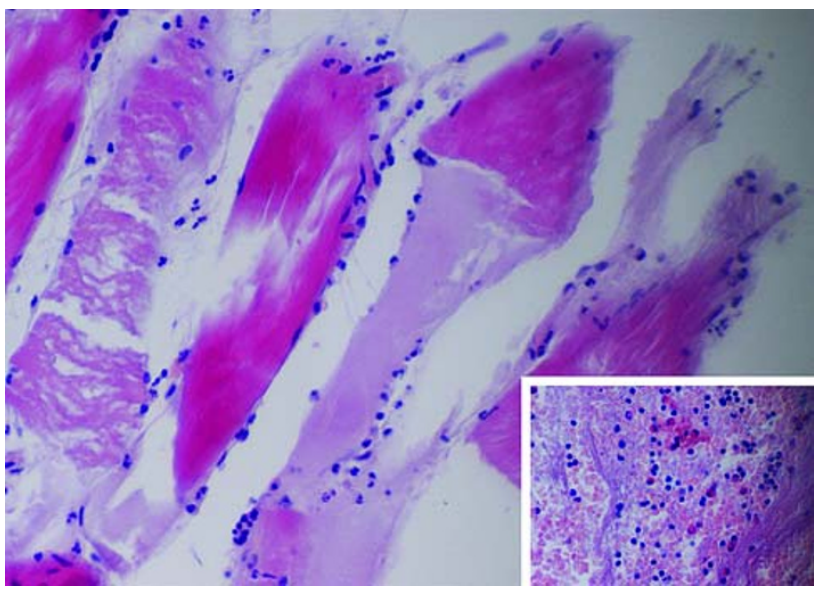

Fig. 1 Histologic findings of muscular tissues in compartment syndrome (H\&E stain). Muscle cells with loss of transverse streaks, atrophy and inflammatory infiltration characterized by neutrophil granulocytes. (original magnification $\times 400$ ). Inset infiltration characterized by lymphocytes, neutrophil and eosinophil granulocytes (original magnification $\times 100$ )

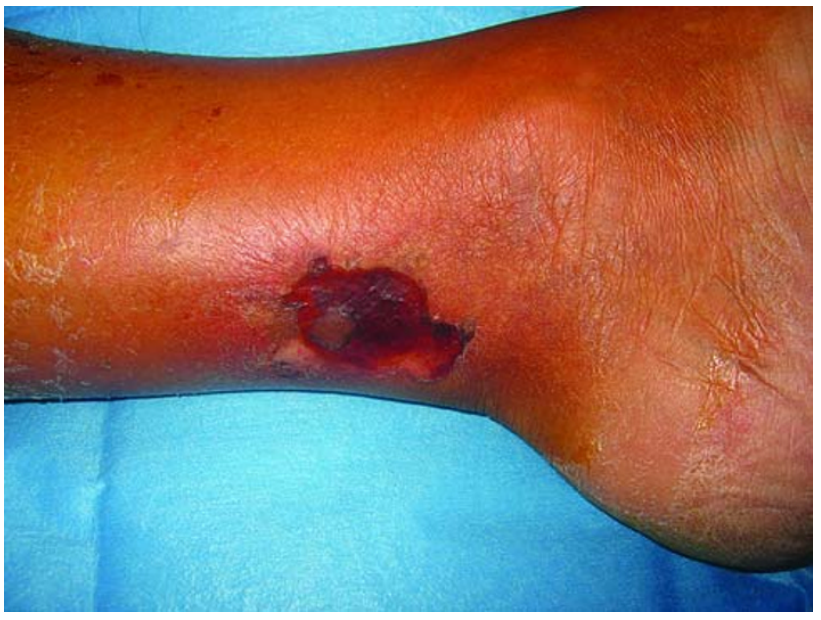

Fig. 2 Ischemic area in the Achilles tendon medial surface then resolved by second-intention healing

evident where the patient had previously complained of pain. The lesion became more evident each day, developing into an ulcer $3 \mathrm{~mm}$ deep and successively resolved by second-intention healing.

We discharged the patient 30 days after surgery and rehabilitation was started immediately. At the first year follow up a decrease of ROM in the ankle was noted, particularly in dorsal flexion, weakness and hypotrophy of the leg were seen.

\section{Discussion}

Traumatic compartment syndrome is a well-known condition and usually easily recognized for its high level of 
suspicion. Otherwise, the Chronic Form is insidious and difficult to diagnose if the patient is not an athlete and there is no temporal correlation with excessive physical stress.

Our case is unusual because it is not very easy to place it in a specific category; the onset was acute but the predisposing factors more typical of chronic forms are easily recognizable. Moreover, the patient was not an athlete and he was at rest during the onset.

The revascularization origin, before being suspected, was excluded by echo-colo-doppler; the biopsy indicated an autoimmunogenic, parasitic or allergic myopathy.

Actually this aspect is still obscure; nevertheless, it was not confirmed by any variation in white blood cells or the presence of self-antibodies and consequently this hypothesis was rejected.

The other hypothesis derived from two specific factors: the diagnosis of type-II Diabetes Mellitus (DM) done during the hospitalization and the three-year administration of atorvastatin.

In literature, we found different descriptions of spontaneous CS associated to DM and to bad glycemic control [13-17]. Nevertheless, these refer to type-I DM; our case referred to type-II and there was no history of uncontrolled glycemia; in addition the diabetes was asymptomatic.

Statins are used for hyperlipidemia therapy. They act by inhibiting HMGCoA reductase, a fundamental enzyme to produce cholesterol-LDL complexes in the liver. We know that they can determine rhabdomyolysis [11] but not manifested CS.

Our case is the first described in literature which includes the contemporaneous presence of the two risk factors. In our case, the administration of atorvastatin compounded the condition already partially compromised by diabetic microangiopathy, followed by rhabdomyolysis and CS. Acute and CCS is a partially unknown, particularly complex situation. If the traumatic forms are easy to diagnose because of the high level of suspicion, the non-traumatic form presents some difficulty that underlines the importance of a correct patient interview. The administration of statins combined with type-II DM activate a vicious circle (inflammation-oedema-necrosis-inflammation), which gives a clear manifestation of compartment syndrome. Our case highlights that a complete and accurate clinical history is essential to make a correct diagnose, thus, facilitating a proper therapy for difficult-to-diagnose cases of CS.
Conflict of interest statement The authors declare that they have no conflict of interest related to the publication of this manuscript.

\section{References}

1. Adornato MC, Glawson S, Sadoff RS (2000) Spontaneous compartment syndrome in a diabetic patient: a case report. J Oral Maxillofac Surg 58(11):1327-1329

2. Chautems RC, Irmay F, Magnin M, Morel P, Hoffmeyer P (1997) Spontaneous anterior and lateral tibial compartment syndrome in a type I diabetic patient: case report. J Trauma 43(1):140-141

3. Dietz BL, Oberg KC (1999) Judicious evaluation of adverse drug reactions: inaccurate assessment of 3-hydroxy-3-methylglutaryl coenzyme A reductase inhibitor-induced muscle injury. Pharmacotherapy 19(2):232-235

4. Godon B, Crielaard JM (2005) Compartment syndrome and sport traumatology. Rev Med Liege 60(2):109-116

5. Hamza KN, Dunkerley GE, Murray CM (1971) Fractures of the tibia. A report of fifty patients treated by intramedullary nailing. J Bone Joint Surg 53-B(4):696-700

6. Jose RM, Viswanathan N, Aldlyami E, Wilson Y, Moiemen N, Thomas R (2004) A spontaneous compartment syndrome in a patient with diabetes. J Bone Joint Surg Br 86(7):1068-1070

7. Matsen F, Winquist R, Krugmire R (1980) Diagnosis and management of compartmental syndromes. J Bone Joint Surg 62A:286

8. Mendieta JM, Rubio J, Elias M, Company R (2004) Bilateral compartment syndrome after surgical perfusion for chronic ischemia of the lower extremities. Rev Esp Anestesiol Reanim 51(3):155-157

9. Olson SA, Glasgow RR (2005) Acute compartment syndrome in lower extremity musculoskeletal trauma. J Am Acad Orthop Surg 13(7):436-444

10. Omar MA, Wilson JP, Cox TS (2001) Rhabdomyolysis and HMG-CoA reductase inhibitors. Ann Pharmacother 35(9):10961107. Review. Erratum in: Ann Pharmacother 35(10):1296

11. Pamoukian VN, Rubino F, Iraci JC (2000) Review and case report of idiopathic lower extremity compartment syndrome and its treatment in diabetic patients. Diabetes Metab 26(6):489-492

12. Parvizi J, Shaughnessy WJ (2002) Compartment syndrome in a patient with familial rhabdomyolysis: a case report. J Bone Joint Surg Am 84-A(11):2046-2049

13. Raso AM, Visentin I, Zan S, Rispoli P, Conforti M, Moniaci D, Ortensio M (2000) Vascular pathology of surgical interest in drug addicts. Minerva Cardioangiol 48(10):287-296

14. Sen S, Chini EN, Brown MJ (2005) Complications after unintentional intra-arterial injection of drugs: risks, outcomes, and management strategies. Mayo Clin Proc 80(6):783-795

15. Silberstein L, Britton KE, Marsh FP, Raftery MJ, D'Cruz D (2001) An unexpected cause of muscle pain in diabetes. Ann Rheum Dis 60(4):310-312

16. Suzuki S, Nonaka A, Kumazawa T (2002) Compartment syndrome in a young male caused by acute alcoholic rhabdomyolysis. Masui 51(10):1127-1128

17. Tischenko GJ, Goodman SB (1990) Compartment syndrome after intramedullary nailing of the tibia. J Bone Joint Surg 72-A(1):41-44 